The Annals of Applied Probability

2001, Vol. 11, No. 4, 1063-1078

\title{
RIGIDITY PERCOLATION AND BOUNDARY CONDITIONS
}

\author{
By ALEXANDER E. HolROyd \\ University of California, Los Angeles
}

\begin{abstract}
We study the effects of boundary conditions in two-dimensional rigidity percolation. Specifically, we consider generic rigidity in the bond percolation model on the triangular lattice. We introduce a theory of boundary conditions and define two different notions of "rigid clusters," called $\mathrm{r}^{0}$-clusters and $\mathrm{r}^{1}$-clusters, which correspond to free boundary conditions and wired boundary conditions respectively. The definition of an $r^{0}$-cluster turns out to be equivalent to the definition of a rigid component used in earlier papers by Holroyd and Häggström. We define two critical probabilities, associated with the appearance of infinite $\mathbf{r}^{0}$-clusters and infinite $\mathrm{r}^{1}$-clusters respectively, and we prove that these two critical probabilities are in fact equal. Furthermore, we prove that for all parameter values $p$ except possibly this unique critical probability, the set of $r^{0}$-clusters equals the set of $\mathrm{r}^{1}$-clusters almost surely. It is an open problem to determine what happens at the critical probability.
\end{abstract}

1. Introduction. Consider the standard bond percolation model, in which each edge of a graph is declared open with probability $p$ and closed otherwise, independently for different edges. The standard theory of percolation focuses on the connectivity properties of the resulting graph of open edges. For details, see [2]. Recently, there has been interest in the study of properties of the graph other than connectivity. Two such properties of particular physical significance are entanglement (see [3, 5, 6]) and rigidity (see [4, 7]).

Our focus here is rigidity. Roughly speaking, a graph is said to be rigid if it cannot be deformed when it is regarded as a structure made up of solid bars and pivots. We shall be concerned with the question of whether the graph of open edges contains large rigid subgraphs. Such questions have important physical applications, in particular to the study of glassy materials; see [8] for details. The combinatorial theory of graph rigidity is also important in its own right; for details see [1].

There are several different concepts of rigidity, but we shall restrict our attention to so-called generic rigidity in two dimensions. We shall give a definition of this in Section 2; for more detailed discussion the reader is referred to $[1,7,8]$. We shall further restrict our attention to percolation on the twodimensional triangular lattice. Many of our techniques would be valid in higher dimensions also, but we shall make use of some results in rigidity theory which are not. The choice of the triangular lattice is a matter of convenience only, and our results could be extended to other planar two-dimensional lattices.

Received March 2000; revised August 2000.

AMS 2000 subject classifications. Primary 60K35; secondary 05B35, 52C25, 82B43.

Key words and phrases. Rigidity percolation, rigidity, percolation, boundary conditions. 
Rigidity is most naturally defined for finite graphs. In [7], the definition for a finite graph was extended in a natural way to infinite graphs. It was proved in [7] that there is a critical probability $p_{\mathrm{r}}$ for the appearance of infinite rigid graphs, and that $p_{\mathrm{c}}<p_{\mathrm{r}}<1$, where $p_{\mathrm{c}}$ is the usual connectivity critical probability. A maximal open rigid graph is called an r-cluster. In [4] it was proved that whenever an infinite r-cluster exists, it is almost surely unique. (A weaker version of this statement was obtained earlier in [7]).

We now discuss the concept of boundary conditions, which has its origins in the theory of statistical physics. The motivation is as follows. Rather than considering infinite rigid graphs in the infinite lattice, we concentrate on a (large) finite portion of the lattice, and try to take the limits of quantities of interest as the size of this finite portion increases. This program presents several challenges. First, which quantities should we consider, and how can we ensure that their limits exist? Second, how should the boundary of the finite portion of the lattice be treated? Different choices of boundary conditions may in principle give different results. We shall develop a detailed theory of boundary conditions for the rigidity percolation model, focusing on the two extremal boundary conditions: "free" boundary conditions, in which we declare all edges outside the finite portion to be closed, and "wired" boundary conditions, in which we declare all such edges to be open.

Aside from its intrinsic mathematical interest, the subject of boundary conditions is of importance in the physical applications of rigidity, and also in numerical simulation (in [8] for example, simulations with two different types of boundary conditions were used to estimate the critical probability and certain exponents). Furthermore, the rigorous study of boundary conditions has the potential to provide new facts relating to other issues, such as continuity of the rigidity percolation probability.

We shall define two different notions of "rigid clusters," called $\mathrm{r}^{0}$-clusters and $\mathrm{r}^{1}$-clusters, which correspond to free boundary conditions and wired boundary conditions, respectively. We shall see that the definition of an $r^{0}$ cluster is in fact equivalent to the definition of an r-cluster mentioned earlier. Thus the previous rigorous studies of rigidity percolation $[4,7]$ can be regarded as treating the free boundary conditions case. In contrast with the situation for connected clusters, where the choice of boundary conditions has only trivial effects, we shall see that there exist configurations for which there is an infinite $r^{1}$-cluster but no infinite $r^{0}$-cluster. (A similar phenomenon occurs in the case of entanglement; see [3]). The question arises whether such configurations can actually occur with positive probability.

We shall define two critical probabilities, for the existence of infinite $\mathrm{r}^{0}$ clusters and infinite $\mathrm{r}^{1}$-clusters respectively. Our main result (Theorem 7) is that these two critical probabilities in fact coincide. Furthermore, we prove (Theorem 14) that at all values of $p$ except possibly the unique critical probability $\left(p_{\mathrm{r}}\right)$, the $\mathrm{r}^{0}$-clusters and the $\mathrm{r}^{1}$-clusters are identical almost surely. (That is, choice of boundary conditions has no effect, except possibly at the critical point). We shall also give some results about continuity of the percolation 
probability functions, and present an open problem concerning their behavior at the critical point $p_{\mathrm{r}}$.

The paper is organized as follows. In Section 2 we first review background material and previous results on rigidity percolation, and then present our main result on boundary conditions. A detailed treatment of boundary conditions is given in Section 3. Much of the material in Section 3 is not used in the subsequent proofs, but helps to demonstrate the significance of the main result. Finally, the main result is proved in Section 4.

2. Background and results. In this section we review previous results in two-dimensional generic rigidity percolation and present the new results to be proved.

We start with some definitions. A graph is pair $G=(V, E)$, where $V$ is a countable set, and $E$ is a set of subsets of size 2 of $V$. The elements of $V$ are called the vertices of $G$, and the elements of $E$ are called the edges of $G$. We shall write $v \in G$ (respectively, $e \in G$ ) to indicate that $v(e)$ is a vertex (edge) of $G$. If $A=\left(V_{A}, E_{A}\right)$ and $B=\left(V_{B}, E_{B}\right)$ are graphs, we define the graphs $A \cup B=\left(V_{A} \cup V_{B}, E_{A} \cup E_{B}\right)$ and $A \cap B=\left(V_{A} \cap V_{B}, E_{A} \cap E_{B}\right)$. We say that $A$ is a subgraph of $B$ if $V_{A} \subseteq V_{B}$ and $E_{A} \subseteq E_{B}$.

The triangular lattice is the graph $\mathbb{T}=(V, E)$ with vertex set

$$
V=\{a(1,0)+b(1 / 2, \sqrt{ } 3 / 2): a, b \in \mathbb{Z}\} \subseteq \mathbb{R}^{2},
$$

and edge set

$$
E=\{\{x, y\} \subseteq V:\|x-y\|=1\},
$$

where $\|\cdot-\cdot\|$ denotes Euclidean distance. The origin is the vertex $O=(0,0) \in$ $V$. We consider the standard bond percolation model on $\mathbb{T}$. That is, for a parameter $p \in[0,1]$, each edge of $\mathbb{T}$ is declared open with probability $p$, and closed otherwise, independently for different edges. More formally, we consider the product probability measure $P_{p}$ on the sample space $\{0,1\}^{E}$. An element $\omega$ of the sample space is called a configuration, and an edge $e$ is open if $\omega(e)=1$ and closed if $\omega(e)=0$. We write $E_{p}$ for the expectation operator associated with $P_{p}$. We write $W=W(\omega)$ for the graph having the same vertex set as $\mathbb{T}$, and whose edge set is the set of all open edges.

The connected components of $W$ are called clusters. In particular, the (connectivity) critical probability $p_{c}$ is defined as

$$
p_{\mathrm{c}}=\sup \left\{p: P_{p}(O \text { lies in an infinite cluster })=0\right\} .
$$

It is a standard result that $0<p_{\mathrm{c}}<1$. See [2] for more details of percolation theory.

We now give a brief treatment of (generic) two-dimensional rigidity of graphs. For a more detailed and general account, see $[1,7]$.

Let $G=(V, E)$ be a finite graph. An embedding of $G$ is an injective map $r: V \rightarrow \mathbb{R}^{2}$. The pair $(G, r)$ is called a framework. A motion of a framework 
$(G, r)$ is a differentiable family $\left(r_{t}: t \in[0,1]\right)$ of embeddings of $G$ with $r=r_{0}$, and such that

$$
\left\|r_{t}(x)-r_{t}(y)\right\| \text { is constant in } \mathrm{t},
$$

whenever $\{x, y\}$ is an edge of $G$. We say that a motion is rigid if (1) holds for all pairs of vertices $x, y \in V$. We say that a framework is rigid if all of its motions are rigid.

The following is a standard result of graph rigidity theory. (See [1] for details.)

PROPOSITION 1. For any finite graph $G$, either $(G, r)$ is rigid for almost all embeddings $r$, or $(G, r)$ is not rigid for almost all embeddings $r$, where "almost all" refers to Lebesgue measure on $\mathbb{R}^{2|V|}$.

We therefore say that a finite graph $G$ is rigid if the framework $(G, r)$ is rigid for almost all embeddings $r$ of $G$.

The next step is to extend the above definition to infinite graphs. The following very natural definition was introduced in [7] and was used subsequently in [4]:

A graph $G$ is said to be rigid if every finite subgraph of $G$ is a subgraph of some rigid finite subgraph of $G$.

(This definition is consistent with the earlier one in the case when $G$ is finite).

The following is a fundamental property of rigid graphs.

Proposition 2. Let $A$ and $B$ be graphs with vertex sets $V_{A}$ and $V_{B}$, respectively.

(i) If $A$ and $B$ are rigid, and $\left|V_{A} \cap V_{B}\right| \geq 2$, then $A \cup B$ is rigid.

(ii) If $\left|V_{A} \cap V_{B}\right| \leq 1$, then $A \cup B$ is not rigid.

For a proof, see [1] or [7]. One simple consequence of Proposition 2(ii) is that every rigid graph is connected.

For any graph $G$, we define an $r$-component of $G$ to be a maximal (in the sense of subgraphs) rigid subgraph of $G$. It is straightforward to show that any isolated vertex of $G$ forms an r-component, and that the edge sets of the r-components of $G$ form a partition of the edge set of $G$ (this follows from Proposition 2). Every vertex of $G$ lies in at least one r-component of $G$, but it may lie in more than one (in contrast with the situation for connected components).

We now return to the percolation model. For a given configuration $\omega$, the r-components of the graph of open edges $W(\omega)$ are called $r$-clusters (of $\omega$ ). For a fixed edge $e$ of $\mathbb{T}$, we define the function

$$
\phi(p)=P_{p}(e \text { lies in an infinite r-cluster }) .
$$


It is easily seen that this probability does not depend on the choice of $e$, and that it is a (nonstrictly) increasing function of $p$. We define the rigidity critical probability $p_{\mathrm{r}}$ by

$$
p_{\mathrm{r}}=\sup \{p: \phi(p)=0\} .
$$

The following was proved in [7].

THEOREM 3. We have

$$
p_{\mathrm{c}}<p_{\mathrm{r}}<1
$$

The following theorems were proved in [4].

THEOREM 4. For all $p$ such that $\phi(p)>0$, there is exactly one infinite r-cluster almost surely.

THEOREM 5. The function $\phi$ is continuous on the interval $\left(p_{\mathrm{r}}, 1\right]$, and $\phi$ is left-continuous or right-continuous (or both) at $p_{\mathrm{r}}$.

(Earlier, in [7], Theorem 4 was obtained for all but countably many values of $p$, and partial progress was made towards Theorem 5).

We now introduce the concept of boundary conditions. We shall give a full treatment in Section 3; here we give only the definitions needed to state our main result.

We start by defining some special subgraphs of $\mathbb{T}$. If $x, y$ are vertices of $\mathbb{T}$, we write $d(x, y)$ for their graph-theoretic distance in $\mathbb{T}$. If $U$ is a set of vertices of $\mathbb{T}$, we write $\mathbb{T}(U)$ for the graph whose vertex set is $U$, and whose edge set consists of all edges of $\mathbb{T}$ having both vertices in $U$. For $n$ a nonnegative integer we define the graph

$$
H_{n}=\mathbb{T}(\{v \in \mathbb{T}: d(O, v) \leq n\}) .
$$

( $H_{n}$ takes the form of a hexagon of side length $n$ centered at the origin). Later, we shall also make use of the set of vertices,

$$
\partial H_{n}=\{v \in \mathbb{T}: d(O, v)=n\},
$$

and the graph

$$
J_{n}=\mathbb{T}(\{v \in \mathbb{T}: n-1 \leq d(O, v) \leq n\}) .
$$

( $J_{n}$ is a kind of "hexagonal annulus" of thickness 1 ).

For $\omega$ a configuration, $n$ a nonnegative integer and $i$ equal to 0 or 1 , we define a new configuration $\omega_{n}^{i}$ by

$$
\omega_{n}^{i}(e)= \begin{cases}\omega(e), & \text { if } e \in H_{n}, \\ i, & \text { if } e \notin H_{n}\end{cases}
$$

We focus on $\omega_{n}^{1}$. (We shall turn our attention to $\omega_{n}^{0}$ in Section 3). It is easy to see that the configuration $\omega_{n}^{1}$ has exactly one infinite r-cluster, and we denote 
it $I_{n}^{1}$. Furthermore, since $W\left(\omega_{n+1}^{1}\right)$ is a subgraph of $W\left(\omega_{n}^{1}\right)$ (for any $\omega$ ), the sequence of graphs $\left(I_{n}^{1}: n \geq 0\right)$ is decreasing. We define the graph

$$
I^{1}=I^{1}(\omega)=\bigcap_{n} I_{n}^{1} .
$$

It is easily seen that $I^{1}$ is either empty or infinite. If $I^{1}$ is infinite, it may be thought of as "the infinite rigid cluster when wired boundary conditions are added." We will show later that the distribution of $I^{1}$ is invariant under automorphisms of $\mathbb{T}$. There are configurations (see Figures 1 and 2) for which $I^{1}$ is infinite but there is no infinite r-cluster. We shall discuss these matters in detail in Section 3.

Let $e$ be a fixed edge of $\mathbb{T}$ incident to $O$. We define

$$
\phi^{1}(p)=P_{p}\left(e \in I^{1}\right) .
$$

Clearly $\phi^{1}$ is an increasing function. We therefore define

$$
p_{\mathrm{r}}^{1}=\sup \left\{p: \phi^{1}(p)=0\right\} .
$$

We shall see in Section 3 that the quantities $\phi(p)$ and $p_{\mathrm{r}}$ already defined arise in a natural way from a consideration of the configurations $\omega_{n}^{0}$ (the "free" boundary conditions case). In the light of this we write $\phi^{0}=\phi$ and $p_{\mathrm{r}}^{0}=p_{\mathrm{r}}$. It is straightforward to show that $\phi^{1} \geq \phi^{0}$, and hence $p_{\mathrm{r}}^{1} \leq p_{\mathrm{r}}^{0}$. We also have the following.

PROPOSITION 6. The function $\phi^{1}$ is right-continuous.

This will be proved below.

We remark that, by using techniques from [4] involving "surrounding circuits," it may be shown that $\phi^{1}$ is left-continuous on $\left(p_{\mathrm{r}}^{1}, 1\right]$, and that if $\phi^{0}(p)>0$ then $\phi^{1}(p)=\phi^{0}(p)$. However, both of these statements (and more) follow from Theorem 7 below, which we shall prove using a different method.

THEOREM 7. We have $p_{\mathrm{r}}^{0}=p_{\mathrm{r}}^{1}$. Furthermore $\phi^{0}(p)=\phi^{1}(p)$ for all $p$ except possibly $p=p_{\mathrm{r}}^{0}=p_{\mathrm{r}}^{1}$.

We therefore revert to the use of the symbol $p_{\mathrm{r}}$ for the unique critical probability $p_{\mathrm{r}}^{0}=p_{\mathrm{r}}^{1}$. In the light of Theorems 5 and 7 and Proposition 6 , exactly one of the following must hold:

(i) $\phi^{0}$ and $\phi^{1}$ are equal everywhere and continuous everywhere.

(ii) $\phi^{0}$ and $\phi^{1}$ are equal everywhere and continuous except at $p_{\mathrm{r}}$, where both are right-continuous.

(iii) $\phi^{0}$ and $\phi^{1}$ are equal and continuous except at $p_{\mathrm{r}}$, where they are unequal, and $\phi^{1}$ is right-continuous while $\phi^{0}$ is left-continuous.

It is a fascinating unsolved problem to determine which of (i)-(iii) is correct. The main step in our proof of Theorem 7 will be the following. 
LEMMA 8. The set $\left\{p: \phi^{1}(p)>\phi^{0}(p)\right\}$ contains no interval of positive length.

Finally in this section we prove Proposition 6 and deduce Theorem 7 from Lemma 8 and results stated earlier.

Proof of Proposition 6. The function $\phi^{1}(p)$ is the decreasing limit as $n \rightarrow \infty$ of $P_{p}\left(e \in I_{n}^{1}\right)$. The event $\left\{e \in I_{n}^{1}\right\}$ depends on the states of only finitely many edges in $\omega$, and so $P_{p}\left(e \in I_{n}^{1}\right)$ is a continuous function of $p$. Thus $\phi^{1}$ is upper semicontinuous and increasing, and hence right-continuous.

Proof of Theorem 7. Since $\phi^{1} \geq \phi^{0}$, Lemma 8 implies immediately that $p_{\mathrm{r}}^{0}=p_{\mathrm{r}}^{1}$. Clearly $\phi^{0}(p)=\phi^{1}(p)$ for $p<p_{\mathrm{r}}$ (since both are zero). Finally if $p>p_{\mathrm{r}}$, combining Lemma 8 with the facts that $\phi^{1} \geq \phi^{0}, \phi^{1}$ is increasing, and $\phi^{0}$ is right-continuous at $p$ (Theorem 5), we obtain $\phi^{0}(p)=\phi^{1}(p)$.

3. Clusters and boundary conditions. The purpose of this section is to develop a theory of boundary conditions for two-dimensional rigidity percolation.

Fix a configuration $\omega$ and recall the definitions of the configurations $\omega_{n}^{i}$ $(i=0,1)$ from Section 2 . For $i$ equal to 0 or 1 , we wish to consider the r-clusters of the configuration $\omega_{n}^{i}$ (that is, the r-components of the graph $W\left(\omega_{n}^{i}\right)$ ), and take some kind of limit as $n \rightarrow \infty$. In order to make sense of this, compare the r-clusters $\omega_{n}^{i}$ with those of $\omega_{n+1}^{i}$. Since the sequence of configurations $\left(\omega_{n}^{0}: n \geq 0\right)$ is increasing, every r-cluster of $\omega_{n}^{0}$ is a subgraph of some r-cluster of $\omega_{n+1}^{0}$. On the other hand, since the sequence $\left(\omega_{n}^{1}: n \geq 0\right)$ is decreasing, every r-cluster of $\omega_{n+1}^{1}$ is a subgraph of some r-cluster of $\omega_{n}^{1}$.

In the light of the above, we make the following definitions. We say that a graph $C^{0}$ is an $r^{0}$-cluster (of $\omega$ ) if there exists an increasing (in the sense of subgraphs) sequence of graphs $\left(C_{n}^{0}: n \geq 0\right)$ such that $C_{n}^{0}$ is an r-cluster of $\omega_{0}^{n}$ for each $n$, and

$$
C^{0}=\bigcup_{n} C_{n}^{0}
$$

(Recall in particular that an isolated vertex of a graph constitutes an r-cluster). We say that a nonempty graph $C^{1}$ is an $r^{1}$-cluster (of $\omega$ ) if there exists a decreasing (in the sense of subgraphs) sequence of graphs $\left(C_{n}^{1}: n \geq 0\right)$ such that $C_{n}^{1}$ is an r-cluster of $\omega_{1}^{n}$ for each $n$, and

$$
C^{1}=\bigcap_{n} C_{n}^{1}
$$

We note that the definition (2) plays no role in the definition of an $\mathrm{r}^{0}$-cluster, because the configuration $\omega_{n}^{0}$ has only finitely many open edges. Definition (2) does play a role in the definition of an $\mathrm{r}^{1}$-cluster, since $\omega_{n}^{1}$ has infinitely many open edges. However, the definition of an $\mathrm{r}^{1}$-component could, if desired, be reformulated in terms of the graph $W\left(\omega_{n}^{1}\right) \cap H_{n+2}$, which has only finitely 
many edges; the use of the infinite graph $W\left(\omega_{n}^{1}\right)$ is a matter of convenience only. Hence (2) is not used in any "essential" way in the definitions of $\mathrm{r}^{i}$ clusters.

In Propositions 9-13 below we present some simple properties of $\mathrm{r}^{i}$-clusters. These propositions hold for every configuration $\omega$. The proofs, all of which are elementary, are deferred until the end of the section.

PRoposition 9. (i) For each of $i=0,1$, every isolated vertex of $W$ forms an $\mathrm{r}^{i}$-cluster, and the edge sets of the $\mathrm{r}^{i}$-clusters form a partition of the edge set of $W$.

(ii) Every $\mathrm{r}^{1}$-cluster is a union of $\mathrm{r}^{0}$-clusters.

The next result states that there is nothing special about the choice of the sequence of graphs $\left(H_{n}: n \geq 0\right)$. Suppose $\left(\hat{H}_{n}: n \geq 0\right)$ is an increasing sequence of finite graphs with the property that $\bigcup_{n} \hat{H}_{n}=\mathbb{T}$, and define an $\hat{\mathrm{r}}^{i}$-cluster analogously to an $\mathrm{r}^{i}$-cluster, but replacing $H_{n}$ with $\hat{H}_{n}$ in the definition of $\omega_{n}^{i}$.

Proposition 10. For any sequence of graphs $\left(\hat{H}_{n}: n \geq 0\right)$ satisfying the above conditions, the set of $\hat{\mathrm{r}}^{i}$-clusters is identical to the set of $\mathrm{r}^{i}$-clusters, for $i=0,1$.

An important consequence of Proposition 10 is that the definition of an $\mathrm{r}^{i}$-cluster is invariant under the symmetries of $\mathbb{T}$. More precisely, let $\Gamma$ be a graph automorphism of $\mathbb{T}$. Then $\Gamma$ induces a natural mapping from subgraphs of $\mathbb{T}$ to subgraphs of $\mathbb{T}$, which we denote also by $\Gamma$. Furthermore, if $\omega$ is a configuration, we define $\Gamma(\omega)$ to be the configuration whose graph of open edges $W(\Gamma(\omega))$ is $\Gamma(W(\omega))$ [that is, $\Gamma(\omega)(e)=\omega\left(\Gamma^{-1} e\right)$ ]. Then we have the following.

Proposition 11. For any graph automorphism $\Gamma$ of $\mathbb{\mathbb { N }}, \Gamma(C)$ is an $\mathrm{r}^{i}$-cluster of $\Gamma(\omega)$ if and only if $C$ is an $\mathrm{r}^{i}$-cluster of $\omega$, for $i=0,1$.

Next we study the properties of $r^{0}$-clusters and $r^{1}$-clusters separately.

PROPOSITION 12. The set of $\mathrm{r}^{0}$-clusters is identical to the set of $\mathrm{r}$-clusters.

Recall the definitions of $I_{n}^{1}$ and $I^{1}$ from Section 2.

PROPOSITION 13. (i) Every finite $\mathrm{r}^{1}$-cluster is an $\mathrm{r}^{0}$-cluster.

(ii) Either (a) $I^{1}$ is empty and there is no infinite $\mathrm{r}^{1}$-cluster or (b) $I^{1}$ is the unique infinite $\mathrm{r}^{1}$-cluster.

Thus, for any configuration, knowledge of the r-clusters and of $I^{1}$ is equivalent to knowledge of the $r^{0}$-clusters and $r^{1}$-clusters. 


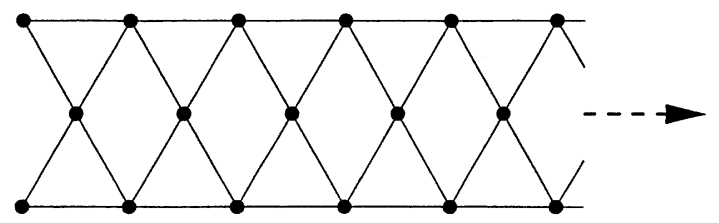

FIG. 1. This configuration has an infinite $\mathrm{r}^{1}$-cluster but no infinite $\mathrm{r}^{0}$-cluster.

In consequence of Propositions 11, 12 and 13, we see that the functions $\phi^{0}$ and $\phi^{1}$ defined in Section 2 may be written as

$$
\begin{aligned}
& \phi^{0}(p)=P_{p}\left(e \text { lies in an infinite } \mathrm{r}^{0} \text {-cluster }\right), \\
& \phi^{1}(p)=P_{p}\left(e \text { lies in an infinite } \mathrm{r}^{1} \text {-cluster }\right),
\end{aligned}
$$

for any edge $e \in \mathbb{T}$.

We note that there exist configurations for which $I^{1}$ is not rigid, for example any configuration having more than one infinite $r$-cluster. Hence $\mathrm{r}^{0}$-clusters and the $r^{1}$-clusters are not necessarily the same. There also exist configurations for which there is an infinite $r^{1}$-cluster but no infinite $r^{0}$-cluster; for example, if $W$ is the infinite graph illustrated in Figure 1, it is easy to check that each triangle is an $r^{0}$-cluster (so that all $r^{0}$-clusters are finite), while there is an infinite $\mathrm{r}^{1}$-cluster containing all the open edges. Can such configurations occur in the percolation model? In Figure 1, although there is no infinite $\mathbf{r}^{0}$ cluster, there exist infinitely many edges $e \in \mathbb{T}$ with the property that adding $e$ to the set of open edges would create an infinite $\mathrm{r}^{0}$-cluster. But it is straightforward to show that for any $p$, the event just described has $P_{p}$-probability zero. On the other hand, in the configuration illustrated in Figure 2, it may be shown that there is an infinite $r^{1}$-cluster but no infinite $r^{0}$-cluster, and that these statements remain true if we change the states of any finite set of edges. The question therefore arises whether configurations such as this can actually occur with positive probability.

Theorem 7 allows us to deduce the following.

THEOREM 14. For every $p$ except possibly $p=p_{\mathrm{r}}$, almost surely with respect to $P_{p}$, the set of $\mathrm{r}^{0}$-clusters is identical to the set of $\mathrm{r}^{1}$-clusters.

PRoOF. If $p<p_{\mathrm{r}}$, almost surely there are only finite $\mathrm{r}^{1}$-clusters, so the required conclusion follows from Proposition 9 and Proposition 13(i). If $p>p_{\mathrm{r}}$, by Proposition 13(i), each finite $r^{1}$-cluster is an $r^{0}$-cluster. There is a unique infinite $\mathrm{r}^{1}$-cluster, $I^{1}$ (Proposition 13 ), and by Theorem $7, I^{1}$ is almost surely equal to the union of all infinite $r^{0}$-clusters. But by Theorem 4 , there is almost surely exactly one infinite $\mathrm{r}^{0}$-cluster. The required conclusion now follows by Proposition 9.

Finally in this section we sketch the proofs of Propositions 9-13. 


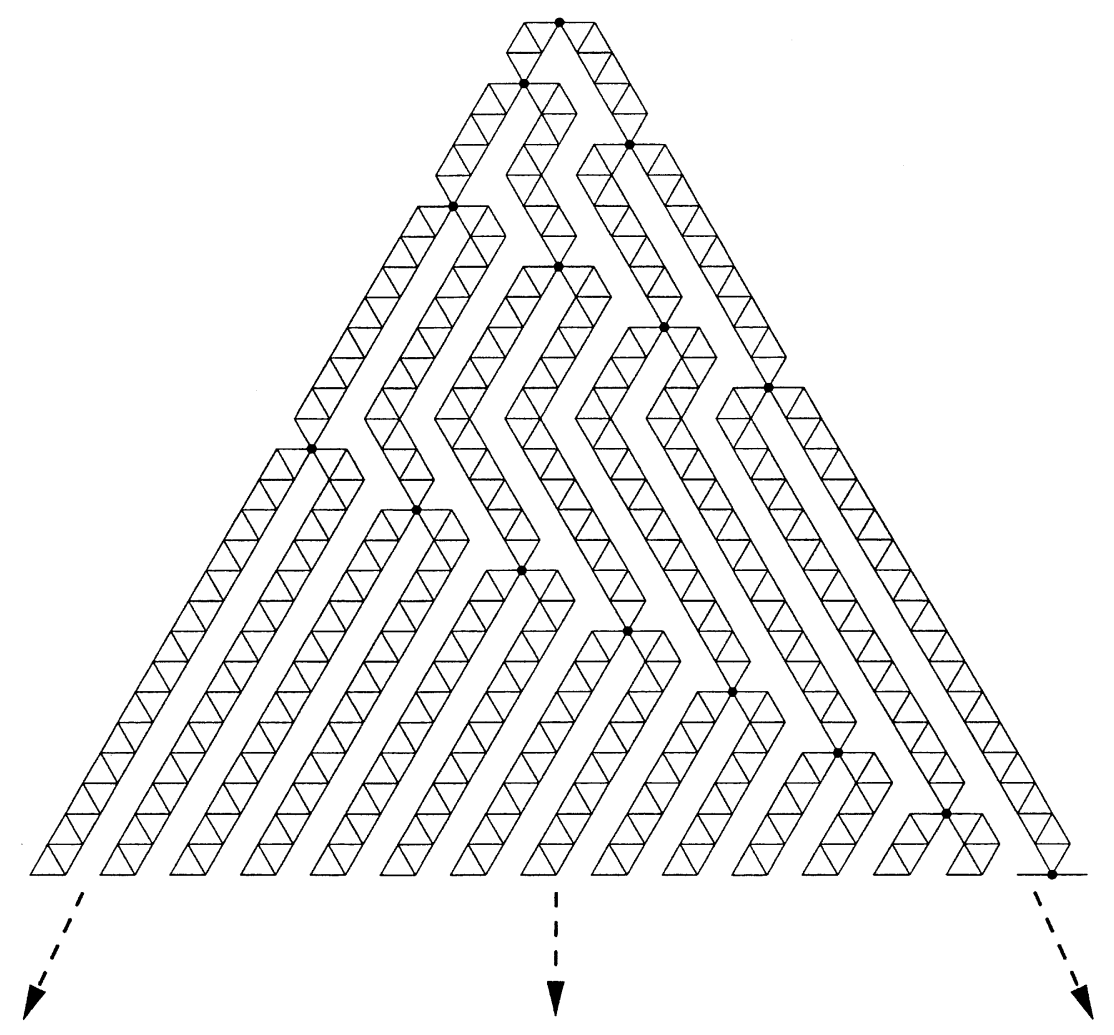

FIG. 2. This configuration has an infinite $\mathrm{r}^{1}$-cluster but no infinite $\mathrm{r}^{0}$-cluster, and these properties are robust to local changes to the configuration.

Proof of Proposition 9. The statements in (i) follow trivially from the corresponding statements for r-components of a graph. For (ii), note that $W\left(\omega_{n}^{0}\right)$ is a subgraph of $W\left(\omega_{n}^{1}\right)$, hence every r-cluster of $\omega_{n}^{0}$ is a subgraph of some r-cluster of $\omega_{n}^{1}$; the result now follows.

Proof of Proposition 10. Let $\left(\hat{H}_{n}: n \geq 0\right),\left(\check{H}_{n}: n \geq 0\right)$ be two sequences of graphs as described, and note that $\left(H_{n}: n \geq 0\right)$ is itself an example of such a sequence. Define an $\check{r}^{i}$-cluster in the obvious way. By the same argument as for $\mathrm{r}^{i}$-clusters, the $\hat{\mathrm{r}}^{i}$-clusters and the $\breve{\mathrm{r}}^{i}$-clusters each partition $W$. We show that for $i=0,1$, any $\hat{\mathrm{r}}^{i}$-cluster is a subgraph of some $\breve{\mathrm{r}}^{i}$-cluster; since by symmetry the same holds with $\hat{\mathbf{r}}^{i}$ and $\breve{\mathrm{r}}^{i}$ reversed, this proves the result. To show that any $\hat{\mathrm{r}}^{1}$-cluster is a subgraph of some $\breve{\mathrm{r}}^{1}$-cluster, we observe that for any $n$, there exists $m$ such that $H_{n}$ is a subgraph of $\hat{H}_{m}$, and hence (with obvious notation) $W\left(\hat{\omega}_{m}^{1}\right)$ is a subgraph of $W\left(\check{\omega}_{n}^{1}\right)$. The argument for the case $i=0$ is similar. 
Proof of Proposition 11. This follows from Proposition 10 on taking $\hat{H}_{n}=\Gamma\left(H_{n}\right)$.

Proof of Proposition 12. If $C^{0}=\cup_{n} C_{n}^{0}$ is an $\mathrm{r}^{0}$-cluster, then it follows immediately from the definition of a rigid graph, (2), that $C^{0}$ is rigid. On the other hand if there exists a rigid subgraph $D$ of $W$ with $C^{0}$ a strict subgraph of $D$, then it is easily seen that there must exist $n$ such that $C_{n}^{0}$ contains edges belonging to $D$ but not to $C^{0}$, giving a contradiction. Thus we have proved that every $\mathrm{r}^{0}$-cluster is an $\mathrm{r}$-cluster, and the converse follows because both the $\mathrm{r}^{0}$-clusters and the r-clusters partition $W$.

Proof of Proposition 13. For (i), suppose $C^{1}=\bigcap_{n} C_{n}^{1}$ is a finite $\mathrm{r}^{1}$-cluster. Then $C^{1}$ is a subgraph of $H_{m}$ for some $m$. Hence, for $n$ sufficiently large, $J_{m+2} \cap C_{n}^{1}$ is empty. But $C_{n}^{1}$ is rigid, and therefore connected, so it follows that for $n$ sufficiently large, $C_{n}^{1}$ is finite. Hence for $n$ sufficiently large, $C_{n}^{1}=C^{1}$. It follows that for all sufficiently large $n, C^{1}$ is an r-cluster of $\omega_{n}^{0}$, so, from the definition of an $\mathrm{r}^{0}$-cluster, $C^{1}$ is an $\mathrm{r}^{0}$-cluster.

We now turn to (ii). It is a triviality that $I^{1}$ is an $\mathrm{r}^{1}$-cluster provided it is nonempty. Now if $C^{1}=\bigcap_{n} C_{n}^{1}$ is an infinite $\mathrm{r}^{1}$-cluster, then each $C_{n}^{1}$ is infinite, so $C_{n}^{1}=I_{n}^{1}$ and hence $C^{1}=I^{1}$. On the other hand, if $I^{1}$ is finite, there must exist $m$ such that $I^{1}$ is a subgraph of $H_{m}$. Hence $J_{m+2} \cap I_{n}^{1}$ is empty for $n$ sufficiently large. But $I_{n}^{1}$ is connected, so this implies that $H_{m} \cap I_{n}^{1}$ is empty for $n$ sufficiently large, and this implies that $I^{1}$ is empty.

4. Main proof. The purpose of this section is to prove Lemma 8. Our proof depends on Lemmas 15-19 below.

LEMMA 15. Let $A, B$ be graphs such that $A \cup B$ is rigid, and let $B^{\prime}$ be a rigid graph with the property that $V_{B^{\prime}} \supseteq V_{A} \cap V_{B}$. Then the graph $A \cup B^{\prime}$ is rigid.

Lemma 15 is proved in [7].

Let $G=(V, E)$ be a finite graph. We introduce the quantity $F(G)$, defined to be the minimum number of extra edges needed to make $G$ rigid:

$$
F(G)=\min \{|X|:(V, E \cup X) \text { is a rigid graph }\} .
$$

We remark that $F(G)$ has physical significance, being exactly three less than the number of "degrees of freedom" or "floppy modes" of $G$. Here is an informal explanation of these terms; for more details see [8]. A graph with no edges has two degrees of freedom for each vertex, corresponding to two possible translations. Adding an edge to a graph may reduce the number of degrees of freedom by one or leave it unaltered. A rigid graph with more than one vertex has three degrees of freedom, corresponding to two translations and one rotation. As we shall see, $F(G)$ also has an interpretation in the combinatorial theory of rigidity (see [1]). 
If $G=(V, E)$ is a graph and $e=\{x, y\}$ is an edge such that $x, y \in V$, we write $G \cup e$ for the graph ( $V, E \cup\{e\}$ ), and $G \backslash e$ for the graph (V,E\\{e\}).

LEMMA 16. Let $G=(V, E)$ be a finite graph and let $e=\{x, y\}$ be an edge such that $x, y \in V$. Then

$$
F(G \cup e)= \begin{cases}F(G), & \text { if } G \text { has an r-component } R \text { with } x, y \in R, \\ F(G)-1, & \text { otherwise. }\end{cases}
$$

The proof of Lemma 16 uses tools from the combinatorial theory of rigidity, and is deferred to the end of this section.

LEMMA 17. If the graphs $A$ and $B$ have the same vertex set $V$, and $A$ is a subgraph of $B$, then for any edge $e=\{x, y\}$ with $x, y \in V$, we have

$$
F(A)-F(A \cup e) \geq F(B)-F(B \cup e) .
$$

We omit the proof of Lemma 17, which is elementary, either directly from the definition of $F$ or from Lemma 16.

Given a configuration $\omega$, we say that an edge $e=\{x, y\}$ of $\mathbb{T}$ is special if the following two statements hold:

(i) $x$ and $y$ are vertices of $I^{1}$.

(ii) There is no r-cluster $C$ with $x, y \in C$.

Note that property (ii) implies that a special edge is closed.

LEMMA 18. If $p$ is such that $\phi^{1}(p)>\phi^{0}(p)$, then for any edge $e$ of $\mathbb{T}$ we have

$$
P_{p}(\text { e is special })>0 .
$$

Our proof of this seemingly obvious lemma is somewhat involved and is deferred to the end of the section.

Our final lemma concerns a kind of "upper integral" for positive functions.

LEMMA 19. Let $a<b$ and let $\sigma:(a, b) \rightarrow(0,1]$ be $a$ positive function. There exists a number $I(\sigma)>0$ such that for every continuously differentiable function $f$ which satisfies

$$
\frac{d}{d x} f(x) \geq \sigma(x) \text { for } x \in(a, b),
$$

we have $f(b)-f(a) \geq I(\sigma)$.

Proof. Define the function $\bar{\sigma}$ on $(a, b)$ by $\bar{\sigma}(x)=\lim _{h \rightarrow 0} \sup _{y \in(x-h, x+h)}$ $\sigma(y)$. The function $\bar{\sigma}$ is upper semicontinuous and therefore Lebesgueintegrable. Furthermore, $\bar{\sigma} \geq \sigma$, and any continuous function $g$ which satisfies $g \geq \sigma$ on $(a, b)$ also satisfies $g \geq \bar{\sigma}$. We therefore define $I(\sigma)=\int_{a}^{b} \bar{\sigma}(x) d x$. 
PROOF OF LEMMA 8. For a given configuration $\omega$ and a positive integer $n$ we define graphs $U_{n}^{i}(\omega)$ for $i=0,1$ as follows. The vertex sets of $U_{n}^{0}$ and $U_{n}^{1}$ are both equal to the vertex set of $H_{n}$. The edges of $U_{n}^{0}$ are the open edges of $H_{n}$, while $U_{n}^{1}=U_{n}^{0} \cup J_{n}$ (recall the definition of $J_{n}$ from Section 2). We think of $U_{n}^{0}(\omega)$ and $U_{n}^{1}(\omega)$ as "finite versions" of $W\left(\omega_{n}^{0}\right)$ and $W\left(\omega_{n}^{1}\right)$, respectively.

The number of edges of $J_{n}$ is bounded above by $k n$ for some fixed constant $k$, and hence we have for each $\omega$,

$$
F\left(U_{n}^{0}\right)-k n \leq F\left(U_{n}^{1}\right) \leq F\left(U_{n}^{0}\right) .
$$

We take expectations and write

$$
f_{n}^{i}(p)=E_{p}\left(F\left(U_{n}^{i}\right)\right)
$$

to give

$$
f_{n}^{0}(p)-k n \leq f_{n}^{1}(p) \leq f_{n}^{0}(p) \text { for all } n \text { and } p .
$$

Note that $f_{n}^{i}(p)$ is a polynomial in $p$ which is decreasing and nonnegative on the interval $[0,1]$.

For a finite graph $G$ and an edge $e=\{x, y\}$ with $x, y \in G$, we say that $e$ is $F$-pivotal for $G$ if

$$
F(G \cup e)=F(G \backslash e)-1 .
$$

[Note that exactly one of $G \cup e$ and $G \backslash e$ is equal to $G$ and that $F(G \cup e)-F(G \backslash e)$ always equals either 0 or -1 ; see Lemma 16.] Using an extension of Russo's formula to random variables (Theorem (2.32) of [2]) we obtain

$$
-\frac{d}{d p} f_{n}^{i}(p)=E_{p} \mid\left\{e \in H_{n}: e \text { is } F \text {-pivotal for } U_{n}^{i}\right\} \mid,
$$

for $i=0,1$.

Since $U_{n}^{0}$ is a subgraph of $U_{n}^{1}$, Lemma 17 implies that any edge which is $F$-pivotal for $U_{n}^{1}$ is also $F$-pivotal for $U_{n}^{0}$. Hence the above equation yields

$$
\begin{aligned}
& -\frac{d}{d p}\left(f_{n}^{0}(p)-f_{n}^{1}(p)\right) \\
& \quad=E_{p} \mid\left\{e \in H_{n}: e \text { is } F \text {-pivotal for } U_{n}^{0} \text { but not for } U_{n}^{1}\right\} \mid,
\end{aligned}
$$

for all $n$.

Next we claim that, for any given configuration $\omega$, if $e$ is a special edge and if $e \in H_{n}$, then $e$ is $F$-pivotal for $U_{n}^{0}$ but not for $U_{n}^{1}$. To prove this, suppose $e=\{x, y\} \in H_{n}$ is a special edge. First, since $W$ has no r-component containing both $x$ and $y, U_{n}^{0}$ has no r-component containing $x$ and $y$ (because $U_{n}^{0}$ is a subgraph of $W$ ). Since $U_{n}^{0} \backslash e=U_{n}^{0}$, this implies by Lemma 16 that $e$ is $F$-pivotal for $U_{n}^{0}$. Second, since $x$ and $y$ lie in $I^{1}$, they lie in some rigid subgraph of the graph $W\left(\omega_{n}^{1}\right)$. Since $W\left(\omega_{n}^{1}\right)$ is obtained from $W$ by adding edges of $\mathbb{T}$ outside $H_{n-1}$, and since $J_{n}$ is a rigid graph containing all the vertices of $\partial H_{n-1}$, an application of Lemma 15 shows that $x$ and $y$ lie in some rigid subgraph of $U_{n}^{1}$. Now if $e \in H_{n} \backslash J_{n}$, then $U_{n}^{1} \backslash e=U_{n}^{1}$, so Lemma 16 implies that $e$ is not $F$-pivotal for $U_{n}^{1}$. On the other hand, it is easy to see that $J_{n}$ remains rigid 
even if any one edge is removed, so if $e \in J_{n}$, then $U_{n}^{1} \backslash e$ has an r-component containing $x$ and $y$, and hence $e$ is not $F$-pivotal for $U_{n}^{1}$ in this case also. We have therefore proved the above claim.

The right-hand side of (4) may now be bounded below by the expected number of special edges in $H_{n}$. By Proposition 11, the probability that an edge is special is the same for all edges, so defining

$$
\sigma(p)=P_{p}(e \text { is special })
$$

for a fixed edge $e$, we obtain

$$
-\frac{d}{d p}\left(f_{n}^{0}(p)-f_{n}^{1}(p)\right) \geq c n^{2} \sigma(p)
$$

for all $n$, where $c$ is a fixed positive constant.

Finally suppose that $\phi^{1}(p)>\phi^{0}(p)$ for all $p \in(a, b)$ where $a<b$. By Lemma 18, $\sigma(p)>0$ for all $p \in(a, b)$. We now appeal to Lemma 19. (Actually, it may be shown that the function $\sigma(p)$ is Lebesgue-integrable, so Lemma 19 is not necessary. However, the argument we give is slightly more concise). We obtain

$$
-f_{n}^{0}(b)+f_{n}^{1}(b)+f_{n}^{0}(a)-f_{n}^{1}(a) \geq c I(\sigma) n^{2}
$$

for all $n$, where $c I(\sigma)>0$. This contradicts (3) when $n$ is large.

Finally, we give the proofs Lemmas 16 and 18. We shall use methods from the combinatorial theory of rigidity; for more information about this see [1]; a brief treatment of some of the relevant material also appears in [7]. We start with some definitions. If $G$ is a graph, the complete graph of $G$ is the graph $K(G)$ which has the same vertex set as $G$, and which has an edge between every pair of vertices.

We define the rigidity closure operator $\langle\cdot\rangle$ as follows. If $G$ is a (finite or infinite) graph, the closure of $G$ is the graph

$$
\langle G\rangle=\bigcup_{H: H \text { is an } \mathrm{r} \text {-component of } G} K(H) .
$$

Note that $\langle G\rangle$ has the same vertex set as $G$, because any vertex of a graph lies in some r-component. The rigidity closure operator has the following properties.

Lemma 20. For any graphs $A, B$ and edges $s, t$ we have:

(i) $\langle A\rangle \supseteq A$.

(ii) If $A \subseteq B$ then $\langle A\rangle \subseteq\langle B\rangle$.

(iii) $\langle\langle A\rangle\rangle=\langle A\rangle$.

(iv) If $s \in\langle A \cup t\rangle$ but $s \notin\langle A\rangle$, then $t \in\langle A \cup s\rangle$ but $t \notin\langle A\rangle$.

Furthermore, it is straightforward to show that $G$ is rigid if and only if $\langle G\rangle=K(G)$. Parts (i)-(iii) of Lemma 20 are reasonably intuitive, but (iv) is perhaps less so. Details of the proof of Lemma 20 may be found in [1], 
and a summary appears in [7]. Actually, the usual approach is to define the rigidity closure operator in a different way, and then prove that both (5) and Lemma 20 hold.

Proof of Lemma 16. For a given finite set $V$ of vertices of $\mathbb{T}$, let $\mathscr{G}$ be the set of all graphs having $V$ as vertex set. Let $K$ be the complete graph with vertex set $V$. The closure operator $\langle\cdot\rangle$ maps $\mathscr{G}$ to itself. The assertions of Lemma 20 are precisely the statement that $\langle\cdot\rangle$ is a matroid closure operator on $\mathscr{I}$ (or, actually, on the edge set of $K$ ). For more details, see [1, 9]. Also, for any graph $G \in \mathscr{G}$, we have that $F(G)$ is the minimum number of edges of $K$ which must be added to $G$ to give a graph whose closure is $K$. A straightforward application of the tools of matroid theory yields that for any matroid closure operator, the function $F$ defined in this way satisfies

$$
F(G \cup e)= \begin{cases}F(G), & \text { if } e \text { is an edge of }\langle G\rangle, \\ F(G)-1, & \text { otherwise, }\end{cases}
$$

for $e$ an edge of $K$; this follows on noting that $F(G)$ is equal to the rank of the matroid minus the rank of $G$; for more details see [1] or [9].

The result now follows from the definition of the closure operator.

Proof of Lemma 18. We shall make use of the rigidity closure operator and of Lemma 20.

Suppose that $\phi^{1}(p)>\phi^{0}(p)$. Then, almost surely with respect to $P_{p}$, the graph $I^{1}$ is infinite and not rigid. Hence almost surely there exist two vertices $u, v$ of $I^{1}$ which do not lie in any one r-cluster. It follows that there exist two fixed vertices $u, v$ of $\mathbb{T}$ such that

$$
P_{p}\left(u, v \in I^{1} \text { but no r-cluster contains } u \text { and } v\right)>0 .
$$

Now, there exists a fixed finite rigid subgraph $R$ of $\mathbb{T}$ with $u, v \in R$ (since $\mathbb{T}$ is rigid). Consider the edge $\{u, v\}$, which is not necessarily an edge of $\mathbb{T}$. On the displayed event above, we have

$$
\{u, v\} \notin\langle W\rangle,
$$

but

$$
\{u, v\} \in\langle W \cup R\rangle
$$

[where for the second statement we have used Lemma 20(i), (ii)]. Hence, writing the edge set of $R$ as $\left\{r_{1}, \ldots, r_{m}\right\}$, there exists $k$ such that

$$
\{u, v\} \notin\left\langle W \cup r_{1} \cup \cdots \cup r_{k-1}\right\rangle,
$$

but

$$
\{u, v\} \in\left\langle W \cup r_{1} \cup \cdots \cup r_{k-1} \cup r_{k}\right\rangle .
$$

Since $u, v \in I^{1}$, we also have that

$$
\{u, v\} \in\left\langle W\left(\omega_{n}^{1}\right)\right\rangle
$$

for each $n$. 
It follows from the above statements that there exist fixed vertices $u, v \in \mathbb{T}$ and a fixed edge $r \in \mathbb{T}$ such that with positive $P_{p}$-probability, the following three statements all hold:

$$
\begin{aligned}
& \{u, v\} \notin\langle W\rangle, \\
& \{u, v\} \in\langle W \cup r\rangle, \\
& \{u, v\} \in\left\langle W\left(\omega_{n}^{1}\right)\right\rangle \quad \text { for all } n .
\end{aligned}
$$

Supposing that (6)-(8) hold, we now apply Lemma 20(iv) to (6) and (7) to deduce that

$$
\begin{aligned}
& r \notin\langle W\rangle \\
& r \in\langle W \cup\{u, v\}\rangle .
\end{aligned}
$$

but

(10)

Finally, combining (10) with (8) and using Lemma 20(i)-(iii) yields

$$
r \in\left\langle W\left(\omega_{n}^{1}\right)\right\rangle \text { for all } n \text {. }
$$

But now (9) and (11) imply that $r$ is a special edge.

Acknowledgments. I thank Lincoln Chayes, Tom Liggett, Roberto Schonmann and Oded Schram for valuable conversations.

\section{REFERENCES}

[1] Graver, J., Servatius, B. and Servatius, H. (1993). Combinatorial Rigidity. Amer. Math. Soc., Providence, RI.

[2] Grimmett, G. R. (1999). Percolation, 2nd. ed. Springer, Berlin.

[3] Grimmett, G. R. and Holroyd, A. E. (2000). Entanglement in percolation. Proc. London Math. Soc. (3) 81, 484-512.

[4] HägGström, O. (2001). Uniqueness in two-dimensional rigidity percolation. Math. Proc. Cambridge Philos. Soc. To appear.

[5] HÄGGSTRÖM, O. (2001). Uniqueness of the infinite entangled component in threedimensional bond percolation. Ann. Probab. To appear.

[6] Holroyd, A. E. (2000). Existence of a phase transition in entanglement percolation. Math. Proc. Cambridge Philos. Soc. 129 231-251.

[7] Holroyd, A. E. (1998). Existence and uniqueness of infinite components in generic rigidity percolation. Ann. Appl. Probab. 8 944-973.

[8] JACobs, D. J. and ThorPe, M. F. (1996). Generic rigidity percolation in two dimensions. Phys. Rev. E 53 3682-3693.

[9] Welsh, D. J. A. (1976). Matroid Theory. Academic Press, New York.

DEPARTMENT OF MATHEMATICS

UNIVERSITY OF CALIFORNIA, LOS ANGELES 405 Hilgard AvENUE

LOS ANGELES, CALIFORNIA 90095-1555

E-MAIL: holroyd@math.ucla.edu 